\title{
Oscillation theorems for second order nonlinear neutral difference equations
}

\author{
Srinivasan Selvarangam ${ }^{1}$, Ethiraju Thandapani ${ }^{2}$ and Sandra Pinelas ${ }^{3 *}$
}

${ }^{\text {*Correspondence: }}$

sandra.pinelas@gmail.com

${ }^{3}$ Departmento de Ciencias Exactas

e Naturais, Academia Militar,

Av. Conde Castro Guimaraes,

Amadora, 2720-113, Portugal

Full list of author information is

available at the end of the article

\section{Abstract}

This paper deals with the oscillation of second order neutral difference equations of the form

$$
\triangle\left(a_{n}\left(\triangle\left(x_{n}+p_{n} x_{\tau(n)}\right)\right)^{\alpha}\right)+a_{n} x_{\sigma(n)}^{\beta}=0
$$

The oscillation of all solutions of this equation is established via comparison theorems. Examples are provided to illustrate the main results.

MSC: $39 \mathrm{~A} 10$

Keywords: neutral difference equation; second order; comparison theorems; oscillation

\section{Introduction}

Consider the second order nonlinear neutral delay difference equation of the form

$$
\triangle\left(a_{n}\left(\triangle\left(x_{n}+p_{n} x_{\mathcal{\tau}(n)}\right)\right)^{\alpha}\right)+q_{n} x_{\sigma(n)}^{\beta}=0, \quad n \geq n_{0} \in \mathbb{N}
$$

where $\mathbb{N}=\{0,1,2, \ldots\}$ and $\triangle$ is the forward difference operator defined by $\Delta x_{n}=x_{n+1}-x_{n}$, subject to the following hypotheses:

$\left(\mathrm{H}_{1}\right) \quad\left\{p_{n}\right\}$ and $\left\{q_{n}\right\}$ are nonnegative real sequences with $\left\{q_{n}\right\}$ not identically zero for infinitely many values of $n$;

$\left(\mathrm{H}_{2}\right)\left\{a_{n}\right\}$ is a positive sequence such that $R_{n}=\sum_{s=n_{0}}^{n-1} \frac{1}{a_{s}^{\frac{1}{\alpha}}} \rightarrow \infty$ as $n \rightarrow \infty$;

$\left(\mathrm{H}_{3}\right) \alpha$ and $\beta$ are ratio of odd positive integers;

$\left(\mathrm{H}_{4}\right)\{\tau(n)\}$ and $\{\sigma(n)\}$ are nondecreasing sequences of integers such that $\lim _{n \rightarrow \infty} \tau(n)=$ $\lim _{n \rightarrow \infty} \sigma(n)=\infty$ and $\tau \circ \sigma=\sigma \circ \tau$;

$\left(\mathrm{H}_{5}\right)$ there is a positive constant $p$ such that $0 \leq p_{n} \leq p<\infty$.

By a solution of equation (1.1) we mean a real sequence $\left\{x_{n}\right\}$ defined, and satisfying equation (1.1), for all $n \geq n_{0}$. We consider only those solutions $\left\{x_{n}\right\}$ of equation (1.1) which satisfy $\sup \left\{\left|x_{n}\right|: n \geq N\right\}>0$ for all $N \geq n_{0}$. We assume that equation (1.1) possesses such a solution. A solution $\left\{x_{n}\right\}$ of equation (1.1) is oscillatory if it is neither eventually positive nor eventually negative, and nonoscillatory otherwise.

Since the second order equations have applications in various problems in physics, biology, and economics, there is a permanent interest in obtaining new sufficient conditions

C2014 Selvarangam et al.; licensee Springer. This is an Open Access article distributed under the terms of the Creative Commons Attribution License (http://creativecommons.org/licenses/by/2.0), which permits unrestricted use, distribution, and reproduction in any medium, provided the original work is properly cited. 
for the oscillation or nonoscillation of solutions of various types of second order equations; see for example [1-12], and the references cited therein.

In $[3,4]$ the authors proved that $0 \leq p_{n}<1$ together with

$$
\sum_{n=n_{0}}^{\infty} q_{n}\left(1-p_{n-\sigma}\right)=\infty
$$

guarantees the oscillation of all solutions of the neutral difference equation

$$
\triangle^{2}\left(x_{n}+p_{n} x_{n-\tau}\right)+q_{n} x_{n-\sigma}=0 .
$$

In [13], the author discussed the oscillatory behavior of all solutions of the difference equation

$$
\triangle\left(a_{n} \triangle\left(x_{n}+p_{n} x_{\tau(n)}\right)\right)+q_{n} f\left(x_{\sigma(n)}\right)=0
$$

with

$$
\frac{f(x)}{x} \geq \mu>0 \quad \text { for } x \neq 0, \quad 0 \leq p_{n}<1 \quad \text { and } \quad \sum_{n=n_{0}}^{\infty} \frac{1}{a_{n}}=\infty
$$

In [14] and [15], the author considered the equation

$$
\triangle\left(a_{n}\left(\triangle\left(x_{n}+p_{n} x_{\tau(n)}\right)\right)^{\alpha}\right)+f\left(n, x_{\sigma(n)}\right)=0
$$

with $\frac{f(n, x)}{x^{\alpha}} \geq c q_{n}$ for $x \neq 0$ and studied the oscillatory behavior under the conditions $\sum_{n=n_{0}}^{\infty} \frac{1}{a_{n}^{\frac{1}{\alpha}}}=\infty$ and $0 \leq p_{n}<1$. In [16], Saker considered the difference equation

$$
\triangle\left(a_{n}\left(\triangle\left(x_{n}+p_{n} x_{n-\tau}\right)\right)^{\alpha}\right)+f\left(n, x_{n-\sigma}\right)=0
$$

with

$$
f(n, x) \geq q_{n} x_{n}^{\alpha}, \quad 0 \leq p_{n}<1 \quad \text { and } \quad \sum_{n=n_{0}}^{\infty} \frac{1}{a_{n}^{\frac{1}{\alpha}}}=\infty
$$

and presented oscillation criteria which improved the existing results for the equation.

In [17], the authors established some sufficient conditions for the oscillation of all solutions of equation (1.3) via comparison theorems. In [18], Sun and Saker considered equation (1.5) and obtained some new oscillation criteria, which improve and complement that given in [16].

Recently in $[19,20]$, the authors studied the oscillatory behavior of all solutions of equation (1.1) under the conditions $0 \leq p_{n} \leq p<1$ and $\alpha>\beta$ or $\alpha<\beta$ or $\alpha=\beta$.

Motivated by the above observation in this paper we shall investigate the oscillatory properties of equation (1.1) without assuming any usual restrictions on $\left\{p_{n}\right\},\{\sigma(n)\}$, and $\{\tau(n)\}$. We shall provide new comparison theorems in which we compare the second order equation (1.1) with the first order difference equations in the sense that the oscillation nature of these first order difference equations yields the oscillation of equation (1.1). In 
Section 2, we present some basic lemmas, and in Section 3 we establish oscillation results. In Section 4, we provide several examples to illustrate the main results and we present our conclusion in Section 5.

\section{Some basic lemmas}

In this section we present some basic lemmas which will be used to prove the main results.

Lemma 2.1 Let $A \geq 0, B \geq 0$, and $\alpha \geq 1$. Then

$$
(A+B)^{\alpha} \leq 2^{\alpha-1}\left(A^{\alpha}+B^{\alpha}\right) .
$$

Proof If $A=0$ or $B=0$, then the result is true. Next, we assume that $0<A \leq B$. Consider the function $f(x)$ defined by $f(x)=x^{\alpha}$. Then

$$
f^{\prime \prime}(x)=\alpha(\alpha-1) x^{\alpha-2} \geq 0
$$

for $x>0$. Therefore, $f(x)$ is convex and $f\left(\frac{A+B}{2}\right) \leq \frac{f(A)+f(B)}{2}$, which implies the result (2.1).

Lemma 2.2 Let $A \geq 0, B \geq 0,0<\alpha \leq 1$. Then

$$
(A+B)^{\alpha} \leq A^{\alpha}+B^{\alpha} .
$$

Proof If $A=0$ or $B=0$, then the result is true. For $A \neq 0$, let $x=\frac{B}{A}$. Then the result (2.2) takes the form $(1+x)^{\alpha} \leq 1+x^{\alpha}$, which is clearly true for all $x>0$.

Next, we present the structure of positive solutions of equation (1.1) since the opposite case is similar.

Lemma 2.3 If $\left\{x_{n}\right\}$ is a positive solution of equation (1.1), then $z_{n}=x_{n}+p_{n} x_{\tau(n)}$ satisfies

$$
z_{n}>0, \quad \triangle z_{n}>0, \quad \triangle\left(a_{n}\left(\triangle z_{n}\right)^{\alpha}\right)<0
$$

eventually.

Proof Assume that $x_{n}>0$ is a solution of equation (1.1). Then equation (1.1) implies

$$
\triangle\left(a_{n}\left(\triangle z_{n}\right)^{\alpha}\right)=-q_{n} x_{\sigma(n)}^{\beta}<0 .
$$

Therefore, $a_{n}\left(\Delta z_{n}\right)^{\alpha}$ is decreasing and thus either $\Delta z_{n}>0$ or $\Delta z_{n}<0$ eventually for $n \geq$ $n_{1} \in \mathbb{N}$. If $\Delta z_{n}<0$, then there exists a negative constant $c$ such that

$$
\triangle z_{n} \leq \frac{c}{a_{n}^{\frac{1}{\alpha}}}<0 .
$$

Summing the above inequality from $n_{1}$ to $n-1$, one obtains

$$
z_{n} \leq z_{n_{1}}+c \sum_{s=n_{1}}^{n-1} \frac{1}{a_{s}^{\frac{1}{\alpha}}} \rightarrow-\infty \quad \text { as } n \rightarrow \infty .
$$

This contradiction proves (2.3). 
The proof of the following lemma is found in [21].

Lemma 2.4 Let $\gamma>1$ be a quotient of odd positive integers. Assume that $k$ is a positive integer, $\left\{d_{n}\right\}$ is a positive sequence defined for all $n \geq n_{0} \in \mathbb{N}$, and there exists $\lambda>\frac{1}{k} \log \gamma$ such that

$$
\lim _{n \rightarrow \infty} \inf \left[d_{n} \exp \left(-e^{\lambda n}\right)\right]>0,
$$

then all the solutions of the difference equation

$$
\triangle y_{n}+d_{n} y_{n-k}^{\gamma}=0
$$

are oscillatory.

Next we state a result given in [22].

Lemma 2.5 Assume that $\left\{\phi_{n}\right\}$ is a nonnegative real sequence for all $n \in \mathbb{N}$ and

$$
\lim _{n \rightarrow \infty} \inf \sum_{j=n-l}^{n-1} \phi_{j}>\left(\frac{l}{l+1}\right)^{l+1}
$$

where $l$ is a positive integer, then

$$
\triangle w_{n}+\phi_{n} w_{n-l} \leq 0
$$

has no eventually positive solution.

Lemma 2.6 Let $\gamma$ be such that $0<\gamma \leq 1$ be a quotient of odd positive integers and $\sigma(n)=$ $n-k$ where $k$ is a positive integer. Assume that $\left\{d_{n}\right\}$ is a positive real sequence defined for all $n \geq n_{0} \in \mathbb{N}$. If

$$
\lim _{n \rightarrow \infty} \inf \sum_{s=n-k}^{n-1} d_{s}>\left(\frac{k}{k+1}\right)^{k+1}
$$

then every solution of the first order delay difference equation

$$
\Delta y_{n}+d_{n} y_{n-k}^{\gamma}=0
$$

is oscillatory.

Proof Assume that $\left\{y_{n}\right\}$ is a positive solution of equation (2.6). First, observe that condition (2.5) implies

$$
\sum_{n=n_{1}}^{\infty} d_{n}=\infty
$$


Since $\left\{y_{n}\right\}$ is decreasing, there exists $l$ such that $\lim _{n \rightarrow \infty} y_{n}=l \geq 0$. If $l>0$, then summing the equation (2.6) from $n_{1}$ to $n-1$, we obtain

$$
y_{n_{1}} \geq \sum_{s=n_{1}}^{n-1} d_{s} y_{s-k}^{\gamma} \geq l^{\gamma} \sum_{s=n_{1}}^{n-1} d_{s} \rightarrow \infty \quad \text { as } n \rightarrow \infty
$$

which is a contradiction, and therefore, we conclude that $\lim _{n \rightarrow \infty} y_{n}=0$, and also $0<y_{n}<$ 1 , eventually. Therefore, $y_{n-k}^{\gamma} \geq y_{n-k}$. Substituting this into the equation (2.6) we deduce that $\left\{y_{n}\right\}$ is a positive solution of the difference inequality

$$
\triangle y_{n}+d_{n} y_{n-k} \leq 0
$$

But this contradicts Lemma 2.5 according to which condition (2.5) ensures that (2.8) has no positive solution. The proof is now complete.

We conclude this section with the following lemma.

Lemma 2.7 Assume that the difference inequality

$$
\triangle w_{n}+\phi_{n} w_{n-k}^{\gamma} \leq 0
$$

has an eventually positive solution, where $\phi_{n} \geq 0, \phi_{n}$ is not identically zero, and $\gamma>0$ is a ratio of odd positive integers. Then the difference equation

$$
\triangle w_{n}+\phi_{n} w_{n-k}^{\gamma}=0
$$

also has an eventually positive solution.

Proof Let $\left\{w_{n}\right\}$ be an eventually positive solution of (2.9). Define a set $S=\left\{u_{n}: 0 \leq u_{n} \leq\right.$ $\left.w_{n}, n \geq N \in \mathbb{N}\right\}$. Then define a mapping $\Gamma$ on $S$ as follows:

$$
(\Gamma u)_{n}= \begin{cases}\sum_{s=n}^{\infty} \phi_{s} u_{s-k}^{\gamma}, & n \geq N, \\ (\Gamma u)_{N}+w_{n}-w_{N}, & n_{0} \leq n \leq N .\end{cases}
$$

Define a sequence $\left\{u_{n}^{(k)}\right\}, k=1,2, \ldots$, as follows:

$$
\begin{aligned}
& u_{n}^{(1)}=w_{n}, \\
& u_{n}^{(k+1)}=\left(\Gamma u^{(k)}\right)_{n}, \quad k=1,2, \ldots
\end{aligned}
$$

Since $\left\{w_{n}\right\}$ is a solution of (2.9), we obtain

$$
\triangle w_{n}+\phi_{n} w_{n-k}^{\gamma} \leq 0
$$

Summing the last inequality from $n$ to $n_{1}$ and letting $n_{1} \rightarrow \infty$, we obtain

$$
-w_{n}+\sum_{s=n}^{\infty} \phi_{s} w_{s-k}^{\gamma} \leq 0
$$


or

$$
u_{n}^{(2)}=(\Gamma w)_{n}=\sum_{s=n}^{\infty} \phi_{s} w_{s-k}^{\gamma} \leq w_{n}=u_{n}^{(1)}
$$

By induction, we see that

$$
0 \leq u_{n}^{(k)} \leq u_{n}^{(k-1)} \leq \cdots \leq u_{n}^{(1)}=w_{n}, \quad n \geq n_{0} .
$$

Hence, $\lim _{k \rightarrow \infty} u_{n}^{(k)}=u_{n}$ exists with $0 \leq u_{n} \leq w_{n}$. Then we can apply the Lebesgue dominated convergence theorem to show that $u=\Gamma u$, that is,

$$
u_{n}=\sum_{s=n}^{\infty} \phi_{n} u_{n-k}^{\gamma}, \quad n \geq N
$$

Clearly, $\left\{u_{n}\right\}$ is an eventually positive solution of (2.10) for all $n \geq N$. Since $u_{n}>0$ for all $n_{0} \leq n \leq N$, it follows that $u_{n}>0$ for all $n \geq n_{0}$. Hence equation (2.10) has an eventually positive solution for all $n \geq n_{0}$. This completes the proof.

\section{Oscillation results}

In this section, we establish some new oscillation criteria for equation (1.1). To simplify our notation, let us denote

$$
Q_{n}=\min \left\{q_{n}, q_{\tau(n)}\right\}, \quad Q_{n}^{\star}=Q_{n}\left(\sum_{s=n_{1}}^{\sigma(n)-1} \frac{1}{a_{s}^{\frac{1}{\alpha}}}\right)^{\beta},
$$

where $n_{1}$ is sufficiently large. We first study the case $\alpha=\beta=1$.

Theorem 3.1 Let $\alpha=\beta=1$ in equation (1.1). Assume that the first order neutral difference inequality

$$
\triangle\left(y_{n}+p y_{\tau(n)}\right)+Q_{n}\left(R_{\sigma(n)}-R_{n_{1}}\right) y_{\sigma(n)} \leq 0
$$

has no positive solution, then every solution of equation (1.1) is oscillatory.

Proof Assume that $\left\{x_{n}\right\}$ is a positive solution of equation (1.1). Then the corresponding function $z_{n}$ satisfies

$$
z_{\sigma(n)}=x_{\sigma(n)}+p_{\sigma(n)} x_{\tau(\sigma(n))} \leq x_{\sigma(n)}+p x_{\sigma(\tau(n))},
$$

where we have used the hypotheses $\left(\mathrm{H}_{4}\right)$ and $\left(\mathrm{H}_{5}\right)$. From equation (1.1), we have

$$
\triangle\left(a_{n} \Delta z_{n}\right)+q_{n} x_{\sigma(n)}=0
$$

and

$$
0=p \triangle\left(a_{\tau(n)} \triangle z_{\tau(n)}\right)+p q_{\tau(n)} x_{\tau(\sigma(n))} .
$$


Combining (3.3) and (3.4), we are led to

$$
\triangle\left(a_{n} \Delta z_{n}+p a_{\tau(n)} \Delta z_{\tau(n)}\right)+Q_{n} z_{\sigma(n)} \leq 0
$$

It follows from Lemma 2.3 that $y_{n}=a_{n} \Delta z_{n}>0$ is decreasing, and then

$$
z_{n} \geq \sum_{s=n_{1}}^{n-1} \frac{1}{a_{s}}\left(a_{s} \Delta z_{s}\right) \geq y_{n} \sum_{s=n_{1}}^{n-1} \frac{1}{a_{s}}=y_{n}\left(R_{n}-R_{n_{1}}\right) .
$$

Therefore, (3.6) together with (3.5) ensures that $\left\{y_{n}\right\}$ is a positive solution of inequality (3.1). This contradicts our assumption and therefore the proof is complete.

Remark 3.1 The condition $\tau \circ \sigma=\sigma \circ \tau$ of the hypothesis $\left(\mathrm{H}_{4}\right)$ is satisfied, for example $\tau(n)=n-\tau$ and $\sigma(n)=n-\sigma$.

Remark 3.2 When studying the oscillatory properties of neutral type equations one usually assumes $\sigma(n) \leq \tau(n), \sigma(n) \leq n, \tau(n) \leq n, 0 \leq p_{n}<1$, etc. In Theorem 3.1 no such constraint is involved and what is more we do not impose is $\tau(n)$ is delayed or advanced and accordingly $\sigma(n)$ can be delayed or advanced. Hence, our result is of high generality and extends and complements the known ones.

Theorem 3.2 Let $\alpha=\beta=1$ in equation (1.1), and assume that

$$
\tau(n) \geq n
$$

If the first order difference inequality

$$
\Delta w_{n}+\frac{Q_{n}}{(1+p)}\left(R_{\sigma(n)}-R_{n_{1}}\right) w_{\sigma(n)} \leq 0
$$

has no positive solution, then every solution of equation (1.1) is oscillatory.

Proof We assume that $\left\{x_{n}\right\}$ is a positive solution of equation (1.1). It follows from Lemma 2.3 and the proof of Theorem 3.1 that $y_{n}=a_{n} \Delta z_{n}>0$ is decreasing and it satisfies (3.1). Let us denote $w_{n}=y_{n}+p y_{\tau(n)}$.

It follows from (3.7) that

$$
w_{n} \leq y_{n}(1+p)
$$

Substituting this term into (3.1), we obtain $\left\{w_{n}\right\}$ is a positive solution of (3.8), a contradiction. This completes the proof.

Adding the restriction that $\sigma(n)=n-\sigma$, and using suitable criterion for the absence of positive solutions of equation (3.8) (see e.g. Lemma 2.5), we obtain an easily verifiable oscillation result for equation (1.1).

Corollary 3.1 Assume that $\alpha=\beta=1$, and (3.7) holds. If

$$
\sigma(n)=n-\sigma, \quad \sigma \text { is a positive integer }
$$


and

$$
\lim _{n \rightarrow \infty} \inf \sum_{s=n-\sigma}^{n-1} Q_{s} R_{\sigma(s)}>(1+p)\left(\frac{\sigma}{\sigma+1}\right)^{\sigma+1},
$$

then every solution of equation (1.1) is oscillatory.

Proof It is easy to see that if (3.10) holds, then

$$
\lim _{n \rightarrow \infty} \inf \sum_{s=n-\sigma}^{n-1} \frac{Q_{s}}{(1+p)}\left(R_{\sigma(s)}-R_{n_{1}}\right)>\left(\frac{\sigma}{\sigma+1}\right)^{\sigma+1} .
$$

But this condition, according to Lemma 2.5, guarantees that (3.8) has no positive solution and the assertion now follows from Theorem 3.2.

Now, we turn our attention to the case when $\tau(n)$ is delayed. We use the notation $\tau^{-1}(n)$ for its inverse function.

Theorem 3.3 Let $\alpha=\beta=1$ in equation (1.1), and assume that

$$
\tau(n) \leq n
$$

If the difference inequality

$$
\triangle w_{n}+\frac{Q_{n}}{(1+p)}\left(R_{\sigma(n)}-R_{n_{1}}\right) w_{\tau^{-1} \sigma(n)} \leq 0
$$

has no positive solution, then every solution of equation (1.1) is oscillatory.

Proof Assume that $\left\{x_{n}\right\}$ is a positive solution of equation (1.1). Then $y_{n}=a_{n} \Delta z_{n}>0$ is decreasing solution of (3.1). We denote $w_{n}=y_{n}+p y_{\tau(n)}$. Then by (3.11), we have $w_{n} \leq$ $y_{\tau(n)}(1+p)$. Substituting this into (3.1), we see that $\left\{w_{n}\right\}$ is a positive solution of inequality (3.12), a contradiction. This completes the proof.

Corollary 3.2 Assume that $\alpha=\beta=1, \tau(n)=n-\tau, \sigma(n)=n-\sigma$ with

$$
n-\sigma \leq n-\tau, \quad \sigma \text { and } \tau \text { are nonnegative integers }
$$

and

$$
\lim _{n \rightarrow \infty} \inf \sum_{s=n+\tau-\sigma}^{n-1} Q_{s} R_{s}>(1+p)\left(\frac{\sigma-\tau}{\sigma-\tau+1}\right)^{\sigma-\tau+1},
$$

then every solution of equation (1.1) is oscillatory.

Proof The proof is very similar to that of Corollary 3.1 and hence it is omitted.

Next, we consider the case $0<\beta \leq 1$, and $\sigma(n) \leq n$ in equation (1.1). 
Theorem 3.4 Let $0<\beta \leq 1$. If the difference inequality

$$
\triangle\left(w_{n}+p^{\beta} w_{\tau(n)}\right)+Q_{n}^{\star} w_{\sigma(n)}^{\frac{\beta}{\alpha}} \leq 0
$$

has no positive solution, then every solution of equation (1.1) is oscillatory.

Proof Let $\left\{x_{n}\right\}$ be a positive solution of equation (1.1). Then from equation (1.1), we have

$$
0=\triangle\left(a_{n}\left(\triangle z_{n}\right)^{\alpha}\right)+q_{n} x_{\sigma(n)}^{\beta}
$$

and

$$
0=p^{\beta} \triangle\left(a_{\tau(n)}\left(\triangle z_{\tau(n)}\right)^{\alpha}\right)+p^{\beta} q_{\tau(n)} x_{\sigma(\tau(n))} .
$$

Combining (3.16) and (3.17), we obtain

$$
\triangle\left(a_{n}\left(\triangle z_{n}\right)^{\alpha}\right)+p^{\beta} \triangle\left(a_{\tau(n)}\left(\triangle z_{\tau(n)}\right)^{\alpha}\right)+Q_{n}\left(x_{\sigma(n)}^{\beta}+p^{\beta} x_{\sigma(\tau(n))}^{\beta}\right) \leq 0 .
$$

By Lemma 2.2, we have

$$
\begin{aligned}
z_{\sigma(n)}^{\beta} & =\left(x_{\sigma(n)}+p_{\sigma(n)} x_{\sigma(\tau(n))}\right)^{\beta} \\
& \leq x_{\sigma(n)}^{\beta}+p^{\beta} x_{\sigma(\tau(n))}^{\beta} .
\end{aligned}
$$

Using (3.19) in (3.18), we obtain

$$
\triangle\left(a_{n}\left(\triangle z_{n}\right)^{\alpha}\right)+p^{\beta} \triangle\left(a_{\tau(n)}\left(\triangle z_{\tau(n)}\right)^{\alpha}\right)+Q_{n} z_{\sigma(n)}^{\beta} \leq 0 .
$$

It follows from Lemma 2.3 that $w_{n}=a_{n}\left(\Delta z_{n}\right)^{\alpha}>0$ is decreasing and so

$$
z_{n} \geq \sum_{s=n_{1}}^{n-1}\left(a_{s}\left(\triangle z_{s}\right)^{\alpha}\right)^{\frac{1}{\alpha}} a_{s}^{-\frac{1}{\alpha}} \geq w_{n}^{\frac{1}{\alpha}} \sum_{s=n_{1}}^{n-1} \frac{1}{a_{s}^{\frac{1}{\alpha}}} .
$$

Using the last inequality in (3.20), we see that $\left\{w_{n}\right\}$ is a positive solution of

$$
\triangle\left(w_{n}+p^{\beta} w_{\tau(n)}\right)+Q_{n}^{*} w_{\sigma(n)}^{\frac{\beta}{\alpha}} \leq 0,
$$

which is a contradiction. This completes the proof.

Next, we shall deduce new sufficient conditions for inequality (3.15) to have no positive solutions, to obtain new oscillation criteria for equation (1.1). We shall discuss both the cases when $\tau(n)$ is delayed and advanced.

Theorem 3.5 Let $0<\beta \leq 1$ and $\tau(n) \geq n$. If the difference equation

$$
\Delta y_{n}+\frac{Q_{n}^{\star}}{\left(1+p^{\beta}\right)^{\frac{\beta}{\alpha}}} y_{\sigma(n)}^{\frac{\beta}{\alpha}}=0
$$

is oscillatory, then so is equation (1.1). 
Proof We assume that $\left\{x_{n}\right\}$ is a positive solution of equation (1.1). Then it follows from the proof of Theorem 3.4 that $w_{n}=a_{n}\left(\Delta z_{n}\right)^{\alpha}>0$ is decreasing and it satisfies (3.15). We denote

$$
y_{n}=w_{n}+p^{\beta} w_{\tau(n)} .
$$

Then

$$
y_{n} \leq w_{n}\left(1+p^{\beta}\right) \text {. }
$$

Substituting this into (3.15), we see that $y_{n}$ is a positive solution of the difference inequality

$$
\Delta y_{n}+\frac{Q_{n}^{\star}}{\left(1+p^{\beta}\right)^{\frac{\beta}{\alpha}}} y_{\sigma(n)}^{\frac{\beta}{\alpha}} \leq 0 .
$$

It follows from Lemma 2.7 that the associated difference equation (3.21) also has a positive solution, which contradicts the oscillatory nature of equation (3.21).

Theorem 3.6 Let $0<\beta \leq 1$ and $\sigma(n) \leq \tau(n) \leq n$. If the difference equation

$$
\Delta y_{n}+\frac{Q_{n}^{\star}}{\left(1+p^{\beta}\right)^{\frac{\beta}{\alpha}}} y_{\tau^{-1}(\sigma(n))}^{\frac{\beta}{\alpha}}=0
$$

is oscillatory, then so is equation (1.1).

Proof Assume that $\left\{x_{n}\right\}$ is a positive solution of equation (1.1). Then it follows from (3.22) that

$$
y_{n} \leq w_{\tau(n)}\left(1+p^{\beta}\right)
$$

or

$$
w_{\sigma(n)}^{\frac{\beta}{\alpha}} \geq \frac{1}{\left(1+p^{\beta}\right)^{\frac{\beta}{\alpha}}} y_{\tau^{-1}(\sigma(n))}^{\frac{\beta}{\alpha}} .
$$

Using the above inequality in (3.15), we see that $\left\{y_{n}\right\}$ is a positive solution of the difference inequality

$$
\Delta y_{n}+\frac{Q_{n}^{\star}}{\left(1+p^{\beta}\right)^{\frac{\beta}{\alpha}}} y_{\tau^{-1}(\sigma(n))}^{\frac{\beta}{\alpha}} \leq 0 .
$$

By Lemma 2.7, the associated difference equation (3.23) also has a positive solution, which is a contradiction. This completes the proof.

Now, we derive a criterion for equations (3.21) and (3.23) to be oscillatory. Employing this criterion, one can easily verify sufficient conditions for the oscillation of all solutions of equation (1.1).

Applying condition (2.6) to equations (3.21) and (3.23) in view of Theorem 3.5 and Theorem 3.6, immediately we obtain the following oscillatory criteria for equation (1.1). 
Corollary 3.3 Let the equation (1.1) with $0<\beta \leq 1, \beta \leq \alpha, \tau(n) \geq n$, and (3.9). If

$$
\lim _{n \rightarrow \infty} \inf \sum_{s=n-\sigma}^{n-1} Q_{s}^{\star}>\left(1+p^{\beta}\right)^{\frac{\beta}{\alpha}}\left(\frac{\sigma}{\sigma+1}\right)^{\sigma+1}
$$

then every solution of equation (1.1) is oscillatory.

Corollary 3.4 Let the equation (1.1) with $0<\beta \leq 1, \beta \leq \alpha, \tau(n)=n-\tau$, and (3.9) with $\sigma>\tau>0$. If

$$
\lim _{n \rightarrow \infty} \inf \sum_{s=n+\tau-\sigma}^{n-1} Q_{s}^{\star}>\left(1+p^{\beta}\right)^{\frac{\beta}{\alpha}}\left(\frac{\sigma-\tau}{\sigma-\tau+1}\right)^{\sigma-\tau+1}
$$

then every solution of (1.1) is oscillatory.

In view of Lemma 2.4, and Theorems 3.5 and 3.6, we have the following oscillation criteria for equation (1.1).

Corollary 3.5 Let the equation (1.1) with $0<\beta \leq 1, \beta>\alpha, \tau(n)=n+\tau$ with $\tau>0$, and (3.9). Assume that there exists $\lambda>\frac{1}{\sigma} \log \frac{\beta}{\alpha}$ such that

$$
\lim _{n \rightarrow \infty} \inf \left[Q_{n}^{\star} \exp \left(-e^{\lambda n}\right)\right]>0
$$

then every solution of equation (1.1) is oscillatory.

Corollary 3.6 Let the equation (1.1) with $0<\beta \leq 1, \beta>\alpha, \tau(n)=n-\tau$, and (3.9) with $\sigma>\tau>0$. Assume that there exists $\lambda>\frac{1}{\sigma-\tau} \log \frac{\beta}{\alpha}$ such that

$$
\lim _{n \rightarrow \infty} \inf \left[Q_{n}^{\star} \exp \left(-e^{\lambda n}\right)\right]>0
$$

then every solution of equation (1.1) is oscillatory.

Next, we turn our attention to the case $\beta \geq 1$ and we rewrite our previous results to cover this case.

Theorem 3.7 Let $\beta \geq 1$ and $\sigma(n) \leq n$. If the difference inequality

$$
\triangle\left(w_{n}+p^{\beta} w_{\tau(n)}\right)+2^{1-\beta} Q_{n}^{\star} w_{\sigma(n)}^{\frac{\beta}{\alpha}} \leq 0
$$

has no positive solution, then every solution of equation (1.1) is oscillatory.

Proof This theorem can be proved exactly as Theorem 3.4; we need to replace the inequality (3.19) by

$$
\begin{aligned}
z_{\sigma(n)}^{\beta} & =\left(x_{\sigma(n)}+p_{\sigma(n)} x_{\tau(\sigma(n))}\right)^{\beta} \\
& \leq 2^{\beta-1}\left(x_{\sigma(n)}^{\beta}+p^{\beta} x_{\sigma(\tau(n))}^{\beta}\right),
\end{aligned}
$$

which follows from Lemma 2.1. 
The following results are equivalent to Theorems 3.5 and 3.6 and the proofs are omitted.

Theorem 3.8 Let $\beta \geq 1$ and $\tau(n) \geq n$. If the difference equation

$$
\Delta y_{n}+\frac{Q_{n}^{\star}}{\left(1+p^{\beta}\right)^{\frac{\beta}{\alpha}}} 2^{1-\beta} y_{\sigma(n)}^{\frac{\beta}{\alpha}}=0
$$

is oscillatory, then every solution of equation (1.1) is oscillatory.

Theorem 3.9 Let $\beta \geq 1$ and $\sigma(n) \leq \tau(n) \leq n$. If the difference equation

$$
\triangle y_{n}+\frac{Q_{n}^{\star}}{\left(1+p^{\beta}\right)^{\frac{\beta}{\alpha}}} 2^{1-\beta} y_{\tau^{-1}(\sigma(n))}^{\frac{\beta}{\alpha}}=0
$$

is oscillatory, then solution of equation (1.1) is oscillatory.

Combining Lemma 2.6 with Theorems 3.8 and 3.9, we have the following oscillation criteria for equation (1.1).

Corollary 3.7 Let $\alpha \geq \beta \geq 1, \tau(n) \geq n$, and $\sigma(n)=n-\sigma$ in equation (1.1). If

$$
\lim _{n \rightarrow \infty} \inf \sum_{s=n-\sigma}^{n-1} Q_{s}^{\star}>2^{\beta-1}\left(1+p^{\beta}\right)^{\frac{\beta}{\alpha}}\left(\frac{\sigma}{\sigma+1}\right)^{\sigma+1}
$$

then every solution of equation (1.1) is oscillatory.

Corollary 3.8 Let $\alpha \geq \beta \geq 1, \tau(n)=n-\tau, \sigma(n)=n-\sigma$ with $\sigma>\tau$ in equation (1.1). If

$$
\lim _{n \rightarrow \infty} \inf \sum_{s=n+\tau-\sigma}^{n-1} Q_{s}^{\star}>2^{\beta-1}\left(1+p^{\beta}\right)^{\frac{\beta}{\alpha}}\left(\frac{\sigma-\tau}{\sigma-\tau+1}\right)^{\sigma-\tau+1}
$$

then every solution of equation (1.1) is oscillatory.

Combining Lemma 2.4 with Theorems 3.8 and 3.9, we have following results.

Corollary 3.9 Let $\beta>\alpha \geq 1, \tau(n) \geq n$, and $\sigma(n)=n-\sigma$ in equation (1.1). Assume that there exists $\lambda>\frac{1}{\sigma} \log \frac{\beta}{\alpha}$ such that

$$
\lim _{n \rightarrow \infty} \inf \left[Q_{n}^{\star} \exp \left(-e^{\lambda n}\right)\right]>0
$$

then every solution of equation (1.1) is oscillatory.

Corollary 3.10 Let $\beta>\alpha \geq 1, \tau(n)=n-\tau, \sigma(n)=n-\sigma$ with $\sigma>\tau$ in equation (1.1). Assume that there exists $\lambda>\frac{1}{\sigma-\tau} \log \frac{\beta}{\alpha}$ such that

$$
\lim _{n \rightarrow \infty} \inf \left[Q_{n}^{\star} \exp \left(-e^{\lambda n}\right)\right]>0,
$$

then every solution of equation (1.1) is oscillatory. 


\section{Examples}

In this section, we present some examples to illustrate the main results.

Example 4.1 Consider the difference equation

$$
\triangle\left(\frac{1}{n} \Delta\left(x_{n}+p x_{\tau(n)}\right)\right)+\frac{b}{n(n+1)} x_{n-2}=0, \quad n \geq 1,
$$

where $0<p<\infty, \tau(n)=a n, a \geq 1$ is an integer and $b>0$. Here $R(n)=\frac{n(n-1)}{2}, Q_{n}=\frac{b}{a n(a n+1)}$. Condition (3.10) reduces to

$$
\frac{27 b}{8 a^{2}}>(1+p)
$$

If the condition (4.2) is satisfied, then by Corollary 3.1, every solution of equation (4.1) is oscillatory.

If $\tau(n)=n-1$, then condition (3.10) reduces to

$$
2 b>(1+p) .
$$

If the condition (4.3) holds, then by Corollary 3.2, every solution of equation (4.1) is oscillatory. Hence, we have covered the oscillation of equation (4.1) when $\tau(n)$ is delayed or advanced.

Example 4.2 Consider the difference equation

$$
\triangle\left(\frac{1}{n}\left(\triangle\left(x_{n}+p x_{\tau(n)}\right)\right)^{\frac{1}{3}}\right)+\frac{b}{n^{\frac{2}{3}}(n+1)^{\frac{2}{3}}} x_{n-2}^{\frac{1}{3}}=0, \quad n \geq 1,
$$

where $0<p<\infty, b>0$, and $\tau(n)=a n, a \geq 1$ is an integer, and $\alpha=\beta=\frac{1}{3}$. Here $R(n)=\frac{n(n-1)}{2}$, $Q_{n}=\frac{b}{(a n)^{\frac{2}{3}}(a n+1)^{\frac{2}{3}}}$. Then condition (3.24) reduces to

$$
\frac{2^{\frac{1}{3}} b}{a^{\frac{4}{3}}}>\frac{8}{27}\left(1+p^{\frac{1}{3}}\right)
$$

Therefore, by Corollary 3.3, every solution of equation (4.4) is oscillatory if condition (4.5) holds.

$$
\begin{aligned}
& \text { If } \tau(n)=n-1 \text {, then condition (3.25) reduces to } \\
& \qquad 2^{\frac{4}{3}} b>1+p^{\frac{1}{3}} .
\end{aligned}
$$

Therefore, by Corollary 3.4, every solution of equation (4.4) is oscillatory if condition (4.6) holds.

Example 4.3 Consider the difference equation

$$
\triangle\left(\frac{1}{n^{\frac{1}{5}}}\left(\triangle\left(x_{n}+p x_{n+1}\right)\right)^{\frac{1}{5}}\right)+\frac{b e^{e^{2 n}}}{(n+1)^{\frac{2}{3}}} x_{n-1}^{\frac{1}{3}}=0, \quad n \geq 1,
$$


where $b>0, \alpha=\frac{1}{5}, \beta=\frac{1}{3}, \tau=1, \sigma=1$. Choose $\lambda=2$, then condition (3.26) reduces to $\frac{b}{2^{\frac{1}{3}}}>0$. Therefore, by Corollary 3.5 every solution of equation (4.7) is oscillatory.

Example 4.4 Consider the difference equation

$$
\triangle\left(\frac{1}{n^{5}}\left(\triangle\left(x_{n}+p x_{\tau(n)}\right)\right)^{5}\right)+\frac{\mu}{(n+1)^{6}} x_{n-2}^{3}=0, \quad n \geq 1
$$

where $\mu>0,0<p<\infty, \alpha=5, \beta=3, \tau(n)=a n, a \geq 1$ is an integer. Here $Q_{n}^{\star}=$ $\frac{\mu}{(a n+1)^{6}}\left(\frac{(n-2)(n-3)}{2}\right)^{3}$. Then condition $(3.31)$ reduces to

$$
\frac{27 \mu}{128 a^{6}}>\left(1+p^{3}\right)^{\frac{3}{5}}
$$

Therefore, by Corollary 3.7, every solution of equation (4.8) is oscillatory if condition (4.9) holds.

Further, if $\tau(n)=n-1$, then condition (3.32) reduces to

$$
\mu>8\left(1+p^{3}\right)^{\frac{3}{5}}
$$

Therefore, by Corollary 3.8, every solution of equation (4.8) is oscillatory if condition (4.10) holds.

\section{Conclusions}

In this paper, we have introduced new comparison theorems for investigation of the oscillation of equation (1.1). The established comparison principles reduce the study of the oscillation of the second order neutral difference equations to a study of the oscillation properties of various types of first order difference inequalities, which clearly simplifies the investigation of the oscillation of equation (1.1). Further, the method used here permits us to relax the restrictions usually imposed on the coefficients of equation (1.1). So the results obtained here are of high generality and easily may be applicable, as illustrated with suitable examples.

\section{Competing interests}

The authors declare that they have no competing interests.

\section{Authors' contributions}

All authors contributed equally to the writing of this paper. All authors read and approved the final manuscript.

\section{Author details}

${ }^{1}$ Department of Mathematics, Presidency College, Chennai, 600005, India. ${ }^{2}$ Ramanujan Institute for Advanced Study in Mathematics, University of Madras, Chennai, 600005 , India. ${ }^{3}$ Departmento de Ciencias Exactas e Naturais, Academia Militar, Av. Conde Castro Guimaraes, Amadora, 2720-113, Portugal.

\section{Acknowledgements}

This paper was supported by CINAMIL - Centro de Investigação, Desenvolvimento e Inovação da Academia Militar.

1. Agarwal, RP: Difference Equations and Inequalities, 2nd edn. Dekker, New York (2000)

2. Elizabeth, S, Graef, JR, Sundaram, P, Thandapani, E: Classifying nonoscillatory solutions and oscillation of neutral difference equations. J. Differ. Equ. Appl. 11, 605-618 (2005)

3. Lalli, BS, Grace, SR: Oscillation theorems of second order delay and neutral difference equations. Util. Math. 45 , 197-212 (1994) 
4. Li, HJ, Yeh, CC: Oscillation criteria for second order neutral difference equations. Comput. Math. Appl. 36, 123-132 (1998)

5. Thandapani, E, Manuel, MMS: Asymptotic and oscillatory behavior of second order neutral delay difference equations. Eng. Simul. 15, 423-430 (1998)

6. Thandapani, E, Mohankumar, P: Oscillation and nonoscillation of nonlinear neutral delay difference equations. Tamkang J. Math. 38, 323-333 (2007)

7. Thandapani, E, Vijaya, M, Gyori, I: New oscillation criteria for forced superlinear neutral type difference equations. Fasc. Math. (to appear)

8. Zhang, BG, Saker, SH: Kamenev-type oscillation criteria for nonlinear neutral delay difference equation. Indian J. Pure Appl. Math. 34(11), 1571-1584 (2003)

9. Agarwal, RP, Manuel, MMS, Thandapani, E: Oscillatory and nonoscillatory behavior of second order neutral delay difference equations. Math. Comput. Model. 24, 5-11 (1996)

10. Agarwal, RP, Wong, PJY: Advanced Topics in Difference Equations. Kluwer Academic, Dordrecht (1997)

11. Agarwal, RP, Bohner, M, Grace, SR, O'Regan, D: Discrete Oscillation Theory. Hindawi Publishing Corporation, New York (2005)

12. Bainov, DD, Mishev, DP: Classification and existence of positive solutions of second order nonlinear neutral difference equations. Funkc. Ekvacioj 40, 371-393 (1997)

13. Jiaowan, L: Oscillation criteria for second order neutral difference equations. Ann. Differ. Equ. 14, $262-266$ (1998)

14. Jiang, J: Oscillatory criteria for second-order quasilinear neutral delay difference equations. Appl. Math. Comput. 125, 287-293 (2002)

15. Jiang, J: Oscillation of second order nonlinear neutral delay difference equations. Appl. Math. Comput. 146, 791-801 (2003)

16. Saker, SH: New oscillation criteria for second order nonlinear neutral delay difference equations. Appl. Math. Comput. 142, 99-111 (2003)

17. Grace, SR, El-Morshedy, HA: Oscillation criteria of comparison type for second order difference equations. J. Appl. Anal. 6, 87-103 (2000)

18. Sun, YG, Saker, SH: Oscillation of second-order nonlinear neutral delay difference equations. Appl. Math. Comput. $163,909-918(2005)$

19. Jinfa, C: Kamenev-type oscillation criteria for delay difference equations. Acta Math. Sci. Ser. B 27(3), $574-580$ (2007)

20. Thandapani, E, Vijaya, M: Oscillation of second order nonlinear neutral delay difference equations. Int. J. Differ. Equ. (to appear)

21. Tang, XH, Liu, Y: Oscillation for nonlinear delay difference equations. Tamkang J. Math. 32, 275-280 (2001)

22. Ladas, G, Philos, CG, Sficas, YG: Sharp condition for the oscillation of delay difference equations. J. Appl. Math. Simul. 2, 101-112(1989)

10.1186/1029-242X-2014-417

Cite this article as: Selvarangam et al.: Oscillation theorems for second order nonlinear neutral difference equations. Journal of Inequalities and Applications 2014, 2014:417

\section{Submit your manuscript to a SpringerOpen ${ }^{\circ}$ journal and benefit from:}

- Convenient online submission

Rigorous peer review

- Immediate publication on acceptance

- Open access: articles freely available online

- High visibility within the field

- Retaining the copyright to your article 